\title{
Internal Shearing within the Hearing Organ Evoked by Basilar Membrane Motion
}

\author{
Anders Fridberger, ${ }^{2 *}$ Jacques Boutet de Monvel, ${ }^{1 *}$ and Mats Ulfendahl ${ }^{1 *}$ \\ ${ }^{1}$ Department of Clinical Neuroscience and Center for Hearing and Communication Research and ${ }^{2}$ Department of \\ Physiology and Pharmacology, Karolinska Institutet, SE-171 76 Stockholm, Sweden
}

The vibration of the hearing organ that occurs during sound stimulation is based on mechanical interactions between different cellular structures inside the organ of Corti. The exact nature of these interactions is unclear and subject to debate. In this study, dynamic structural changes were produced by stepwise alterations of scala tympani pressure in an in vitro preparation of the guinea pig temporal bone. Confocal images were acquired at each level of pressure. In this way, the motion of several structures could be observed simultaneously with high resolution in a nearly intact system. Images were analyzed using a novel wavelet-based optical flow estimation algorithm. Under these conditions, the reticular lamina moved as a stiff plate with a center of rotation in the region of the inner hair cells. Despite being enclosed in several types of supporting cells, the inner hair cells, together with the adjacent inner pillar cells, moved in a manner signifying high compliance. The outer hair cells displayed radial motion indicative of cellular bending. Together, these results show that shearing motion occurs between several parts of the organ, and that structural relationships within the organ change dynamically during displacement of the basilar membrane.

Key words: cochlear mechanics; basilar membrane; outer hair cells; cellular bending; pressure changes; mechanical properties
Hearing is based on an interaction of mechanical and electrical events that occur in the organ of Corti in the auditory part of the inner ear. The organ of Corti is located in the scala media, with its upper surface (the reticular lamina) facing the scala vestibuli and its base (the basilar membrane) toward the scala tympani. When sound reaches the ear, it causes vibration of the tympanic membrane and the middle ear ossicles. The vibrations of the stapes produce pressure gradients between the fluid-filled compartments of the cochlea. As a result of the pressure gradient, the basilar membrane and the hearing organ are shifted repetitively between the scala vestibuli and scala tympani.

The characteristics of this motion are determined primarily by the mechanical properties (stiffness, mass, and friction) of the elements that compose the organ of Corti. These basic mechanical properties are unknown to a significant extent, although direct measurements are available for several of the components of the organ (von Békésy, 1960; Flock and Strelioff, 1984; Zwislocki and Cefaratti, 1989; Olson and Mountain, 1994; Russell and Schauz, 1995; Tolomeo et al., 1996; Tolomeo and Holley, 1997; Chan et al., 1998; Naidu and Mountain, 1998; Ulfendahl et al., 1998). Defining the mechanical properties of the individual elements is undoubtedly of interest, but it is also of fundamental importance to obtain a more integrated understanding of how the

\footnotetext{
Received May 20, 2002; revised Aug. 19, 2002; accepted Aug. 27, 2002.

This work was supported by the Swedish Research Council, the Tysta Skolan Foundation, the National Association for Hard of Hearing People, the Swedish Council for Working Life and Social Research, the Petrus and Augusta Hedlund Foundation, the Tore Nilson Foundation, and funds from the Karolinska Institutet, the Royal Academy of Sciences, and in part the European Commission (QLG3-CT2000-01343). Per Conradi is acknowledged for participation in early experiments.

*All authors contributed equally to this paper.

Correspondence should be addressed to M. Ulfendahl, Centrum för Hörsel och kommunikationsforskning, M1, Karolinska Sjukhuset, SE-171 76 Stockholm, Sweden. E-mail: mats.ulfendahl@cfh.ki.se.

Copyright (C) 2002 Society for Neuroscience $\quad 0270-6474 / 02 / 229850-08 \$ 15.00 / 0$
}

structures interact when subjected to the mechanical forces related to sound stimulation.

Using an in vitro preparation consisting of a cochlea cut in half, $\mathrm{Hu}$ et al. (1999) stimulated the basilar membrane with a vibrating probe while observing the motion of the organ with video microscopy. Their measurements were restricted to low frequencies and to point-like stimulation of the organ but nonetheless provided interesting information about the interaction of different structures, although in a relatively disrupted preparation of the cochlea. Multidimensional vibration data acquired during sound stimulation have been presented by Ulfendahl et al. (1995) and by Gummer et al. (1996) (see also Hemmert et al., 2000a). Although aspects of these studies are still debated, it seems clear that sound evokes a shearing motion between the reticular lamina and the tectorial membrane, as predicted by ter Kuile (1900) purely on the basis of anatomical observations. Obviously, there is need for additional experimental data regarding interactions between different structures within the organ, in particular concerning the motion pattern of the basilar membrane, hair cell bodies, and supporting cells. In the present study, we attempted to experimentally mimic the movements of the hearing organ during sound stimulation by applying stepwise pressure gradients across the cochlear partition to shift the position of the basilar membrane in a controlled manner. Using an in vitro preparation of the guinea pig cochlea and laser-scanning confocal microscopy, we were able to characterize the motion of individual cellular elements within the organ of Corti. In combination with advanced image analysis algorithms, this approach provided an opportunity to investigate the relative motion of different cochlear structures with high resolution in a nearly intact system.

\section{MATERIALS AND METHODS}

Young pigmented guinea pigs weighing 300-450 gm were decapitated, and the temporal bones were excised rapidly and attached to a plastic holder on the bottom of a chamber containing minimum essential me- 


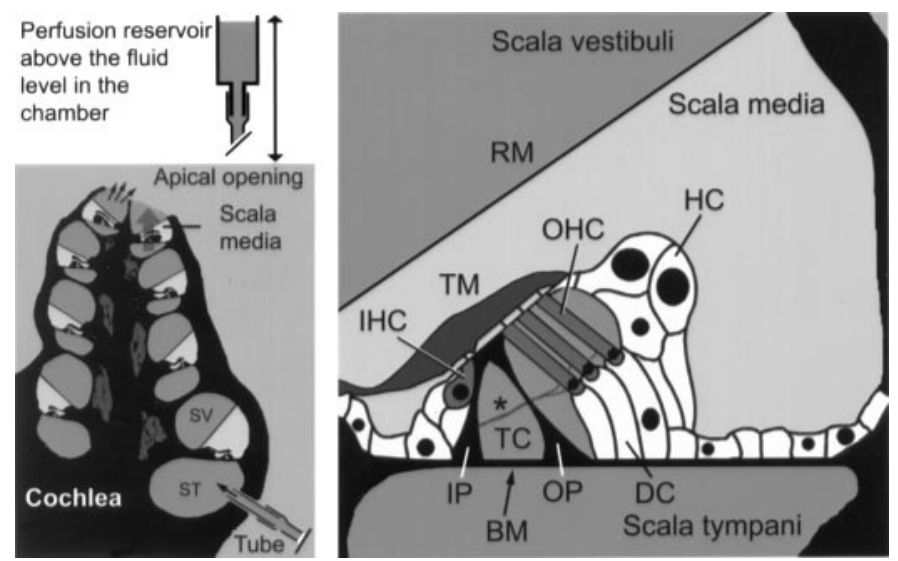

Figure 1. Schematic drawing of the insertion of the perfusion tube into the scala tympani $(S T)$ of the basal turn (left). Fluid flowed continuously through the scala tympani to exit through the helicotrema (small arrows). An increase in scala tympani pressure shifted the organ in the direction of the scala vestibuli ( $S V$; large arrow). Fluid compartments filled with perilymph are dark gray; endolymphatic compartments are light gray. Right, Anatomical structures visible in subsequent figures are indicated in this schematic drawing of a cross section of the organ of Corti in the apical turn. $B M$, Basilar membrane; $D C$, Deiter cell; $O P$, outer pillar cell; $T C$, tunnel of Corti; $I P$, inner pillar cell; $I H C$, inner hair cell; $T M$, tectorial membrane; $O H C$, outer hair cell; $H C$, Hensen cell; $R M$, Reissner's membrane. The asterisk indicates a nerve fiber crossing the tunnel of Corti to reach the outer hair cells.

dium with HBSS and 25 mm HEPES (Invitrogen, Paisley, UK). The medium was bubbled continuously with oxygen and maintained at 22$25^{\circ} \mathrm{C}$ throughout the experiment. After the middle ear cavity had been exposed, a window was made in the apical turn of the cochlea using a fine needle. A second hole was drilled carefully in the scala tympani of the basal turn, and a plastic tube attached to a reservoir containing tissue culture medium was inserted. The medium flowed through the tube into the scala tympani to exit through the apical opening. Using the perfusion system, the fluorescent dyes calcein AM (final concentration $60 \mu \mathrm{g} / \mathrm{ml}$, stock solution dissolved in ethanol) and RH795 (40-50 $\mu \mathrm{g} / \mathrm{ml}$; DMSO) were applied to the preparation. Both dyes came from Molecular Probes (Eugene, OR). These dyes label the cytoplasm and membranes of both supporting cells and sensory hair cells.

Pressure changes. To shift the position of the basilar membrane, the pressure in the scala tympani was modified using the perfusion system (Fig. 1). By moving the fluid-filled perfusion reservoir to positions above the fluid level of the preparation chamber, the scala tympani pressure increased and the cochlear partition shifted upward. Moving the reservoir below the preparation chamber reversed the pressure gradient and shifted the partition downward. Each position was maintained for $20 \mathrm{sec}$ to allow the confocal microscope to acquire an image before moving on to the next pressure level. An experiment usually consisted of a cycle of several levels of both positive and negative pressure, with the acquisition of an image at each pressure. The pressure reached within the scala tympani in the apical turn is related linearly to the height of the fluid column in the tubing connecting the reservoir and the chamber containing the preparation, but the absolute pressure cannot be calculated directly, because the exact impedance of the cochlear segments before the apical turn is unknown. The dimensions of the scala tympani in the apical turn are very small (Voie et al., 1993), and direct measurements of scala tympani pressures are therefore difficult. Moreover, a certain amount of fluid leakage will be present around the insertion of the tube into the cochlea, and this will further limit the pressure acting on the structures in the apical turn. A detailed description of factors affecting the pressure levels in the apical turn has been published previously (Fridberger et al., 1997). From the measurements performed by Fridberger et al. (1997), it was evident that the maximum pressure reached in the scala tympani of the apical turn was $<1 \mathrm{~Pa}$, the limit of sensitivity of the pressure probe used during those experiments. During experiments, the perfusion pressure was kept slightly positive between image acquisitions to ensure oxygenation of the preparation. This may have created a small bias on the cochlear partition that may have contributed to the asymmetric motion described below.
Confocal microscopy. The chamber containing the preparation was mounted on the stage of a Bio-Rad (Hemel Hempstead, Hertfordshire, UK) MRC1024 confocal microscope, the fluorescent dyes were excited using the 488 and $568 \mathrm{~nm}$ lines of a $\mathrm{Kr} / \mathrm{Ar}$ laser, and the cochlear structures were viewed using a Zeiss (Jena, Germany) $40 \times$ numerical aperture 0.75 lens. A full description of the use of confocal microscopy on this preparation has been published previously (Flock et al., 1999; Ulfendahl et al., 2000). Images were acquired using a $768 \times 512$ pixel format, the green fluorescence of the calcein stain was directed to one photomultiplier via a dichroic mirror, and the longer-wavelength fluorescence from the RH795 stain was detected by a second photomultiplier. Either four or five sequential frames were averaged using the Kalman filter built into the Bio-Rad confocal control software, with the iris diaphragm, gain, and laser power adjusted to acquire images with optimum contrast. In preparations with weak fluorescence, the size of the iris diaphragm had to be increased to get satisfactory intensity. Images were saved to disk, and initial processing was done via custom macros running under the freeware image analysis packages Scion Image or ImageJ. Later, Matlab routines were developed to calculate the motion of the structures affected by the pressure changes (see below). Fifteen different preparations provided data for this study. In 10 of those, relatively large pressure amplitudes were used, but this produced images in which most of the cochlear structures moved, creating problems with finding a fixed reference point for comparing the motion of different structures. Five experiments were performed using lower amplitudes, and in this series of

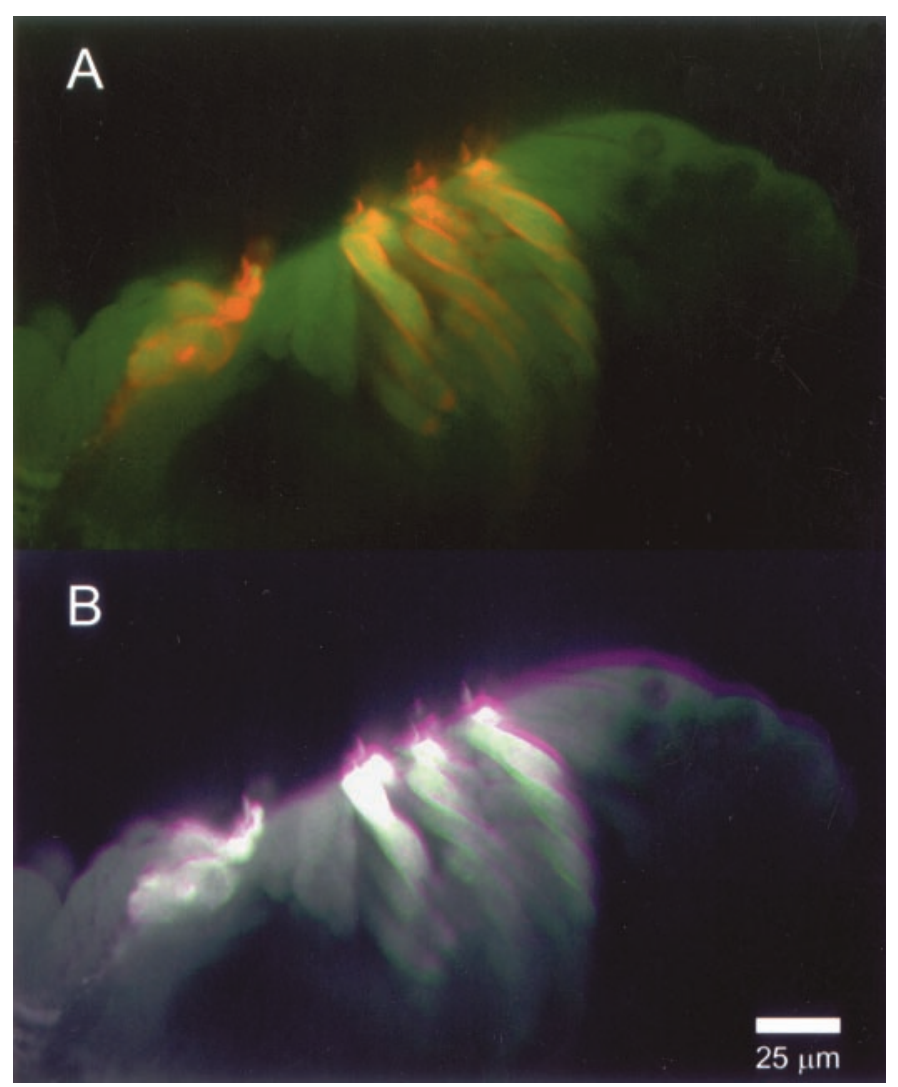

Figure 2. A, Confocal view of the hearing organ in the apical turn. Red staining originates from the membrane dye RH795, and green stain originates from the cytoplasmic dye calcein. $B$, Merged image of the organ at two different pressure levels. The image was generated through averaging the red and green channels of the RGB image shown in $A$ to form a single-channel grayscale image. This grayscale image was subsequently given a pink color. Similar averaging was applied to an image acquired at a different pressure level, and that image was coded green. Thus, structures that overlap each other in the two images will appear gray, and structures that moved will be either pink or green. Note the relatively small motion in the inner hair cell region and the graded increase in amplitude that occurred when moving to the right part of the image. The scale bar applies to both panels. 
Figure 3. $A$, High-magnification view of the tunnel of Corti region. $B$, Merged RGB image of the same part of the organ, generated by applying the same scheme as in Figure $2 B$ (image at high pressure, pink; image after decrease in pressure, green). The scale bar applies to both panels.

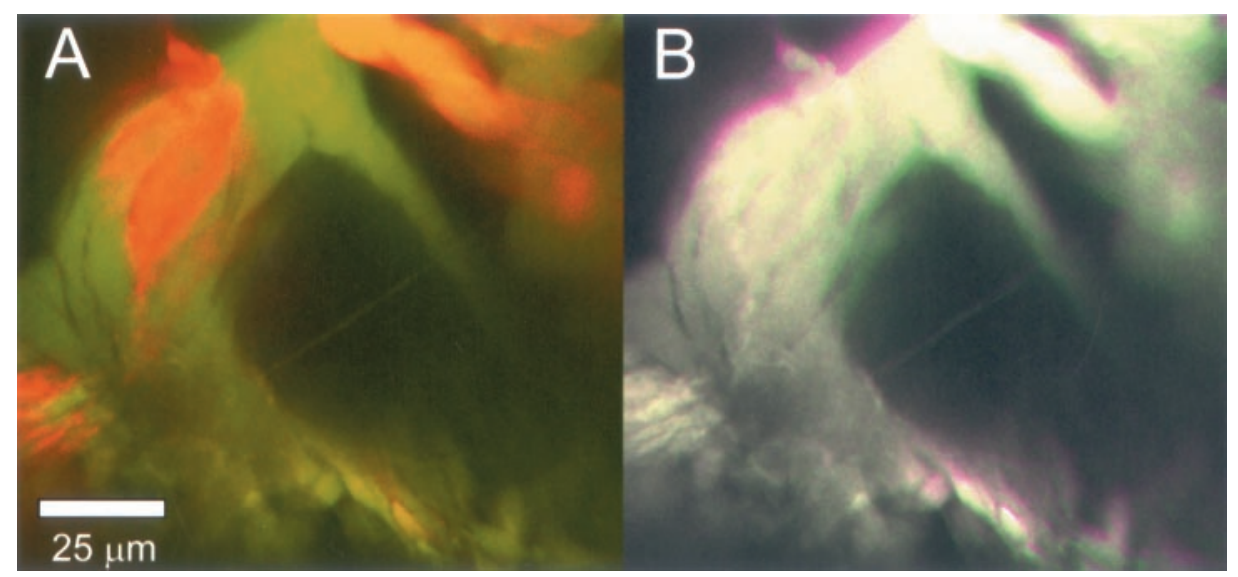

experiments, the nerve fibers under the inner hair cells could often be used as reference points, because they did not show any appreciable motion. These five preparations were analyzed in detail by use of the algorithm described below.

Motion analysis. A differential optical flow technique was used to measure the movement patterns of the organ. This approach is based on the assumption that the intensity of any given point of the sample remains constant along its trajectory in image plane. One may then use a motion constraint equation (Horn and Schunck, 1981): $\partial_{t} I(\mathbf{x}, t)+\mathbf{v}(\mathbf{x}, t)$. $\nabla I(\mathbf{x}, t)=0$, where $I(\mathbf{x}, t)$ is the image intensity, $\partial_{t} I(\mathbf{x}, t)$ and $\nabla I(\mathbf{x}, t)$ denote the image temporal derivative and two-dimensional spatial gradient, respectively, and $\mathbf{v}(\mathbf{x}, t)$ is the unknown velocity vector of a point $\mathbf{x}=(x, y)$ at time $t$. The dot in this equation denotes the usual scalar product of vectors of the plane. The above constraint holds only approximately for confocal image sequences, but the approximation was found to be good enough to allow reliable motion estimation. To circumvent the aperture problem (Barron et al., 1994), we used a multiscale technique inspired by the method of Bernard (2001), which consists of first filtering the image sequence by transforming each frame with a nondecimated twodimensional wavelet transform. Applying the above equation to all wavelet components then yields an overdetermined linear system for $\mathbf{v}(\mathbf{x}, t)$, which is solved by least-squares inversion. A smoothing of the image sequences by the wavelet denoising method described by Boutet de Monvel et al. (2001) was used before the estimation. The time and space derivatives of the wavelet-filtered images were estimated by simple and central differences, respectively. The method was tested on confocal image sequences with computer-generated displacements. Displacements on the order of the pixel size $\sim 0.3 \mu \mathrm{m}$ were measured with $\leq 3 \%$ error; displacements $\leq 0.33$ or $\geq 3$ pixels were measured with $10 \%$ error. For very small displacements, the precision degraded abruptly, setting the lower detection limit to 0.05 pixels.

\section{RESULTS}

\section{General features of organ of Corti motion}

A typical confocal microscope image of the hearing organ is shown in Figure $2 \mathrm{~A}$. Most functionally relevant structures were visible, with the exception of the tectorial membrane, which was not stained by the present set of vital dyes (Ulfendahl et al., 2000). Both inner and outer hair cells with their stereocilia were seen readily along with the different types of supporting cells. A section of the basilar membrane, forming the bottom of the tunnel of Corti, was visible. The nerve endings contacting the inner hair cells were also labeled in many experiments.

The effects of applying variable pressure gradients across the cochlear partition were investigated by acquiring consecutive confocal microscope images of the same region of the hearing organ. As expected, an increase in the scala tympani pressure caused a motion of the organ of Corti toward the scala vestibuli, and a decrease in the pressure moved the organ in the direction of the scala tympani (compare Fig. 1). Figure $2 B$ shows a combined image of the organ before a pressure gradient was applied (in pink; the same image as in Fig. $2 A$ ) and after the scala tympani pressure had been reduced (in green; regions in which the structures overlapped will be seen as gray). There was little displacement in the regions of the organ close to the center of the cochlea, whereas more peripheral structures moved progressively more, with maximum amplitude seen in the Hensen's cell region.

Figure $3 A$ shows the tunnel of Corti region in another preparation at high magnification. Note the intense labeling of the hair cells and nerve fibers, including the fiber crossing the tunnel of Corti and the space of Nuel. From the combined red-green-blue (RGB) image (Fig. 3B), it was evident that the motion of this region of the organ was relatively small, and that the region under the inner hair cells showed negligible motion. Images such as the ones shown above provide a qualitative estimate of organ of Corti motion, especially when viewed as an animated image sequence. Examples of such sequences can be viewed or downloaded at www.ki.se/cfh/movies.

\section{Analysis of cellular motion using optical flow}

To better characterize the displacement of different cochlear structures, an optical flow estimate was applied to the image sequences. Figure 4 shows the trajectory plots obtained by following the displacements of several points along the reticular lamina (Fig. $4 a-d$ ), on the bodies of inner and outer hair cells (Fig. $4 e-h$ ), and on the inner and outer pillar cells (Fig. $4 i-l$ ). At these low frequencies, the organ of Corti showed quasi-static motion, without any detectable phase differences between the motions of the inner and outer hair cells or of any other structure. However, the different structures of the organ followed very different motion patterns. The reticular lamina did not show significant radial motion (Fig. $4 a-d$ ), but a clear radial displacement was observed for the cell bodies of the outer hair cells and the outer pillar cell during the first phase of motion, when the organ was displaced toward the scala vestibuli (Fig. $4 g, h, l$ ). Also, the top and bottom parts of the inner pillar cell followed differently oriented trajectories, with the orientation becoming more radial in the bottom parts (Fig. 4i-k), indicating inner pillar cell deformation.

The amplitudes of the trajectories along the reticular lamina grew nearly linearly from the inner hair cell apex to the third-row outer hair cell (Fig. 4, top right), a feature compatible with a rotation of the reticular lamina around an axis located in the inner hair cell region. The amplitudes are larger than those found during sound stimulation, but these images nonetheless reveal interesting information about the mechanical properties of the organ and the interactions between different structures. 

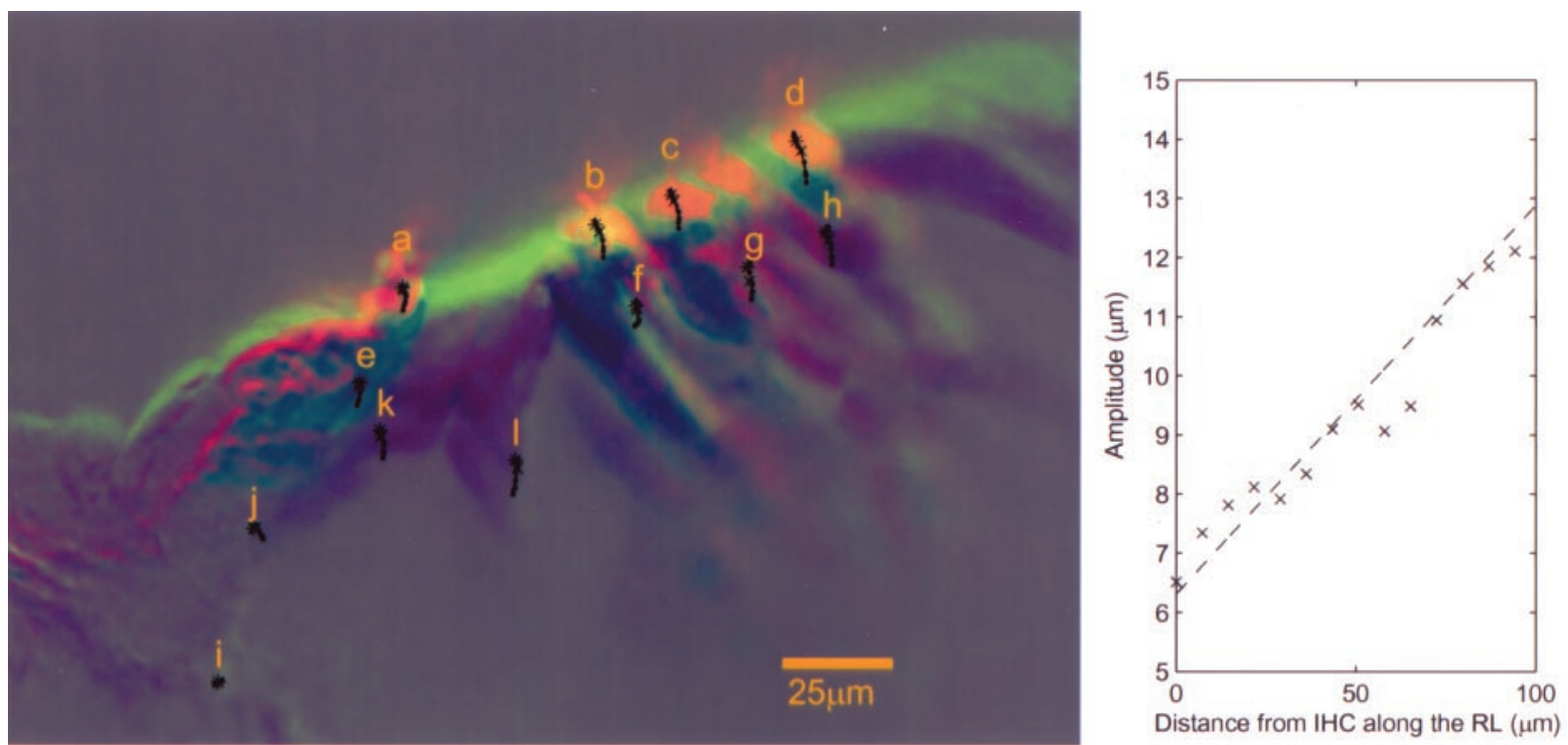

a) IHC top
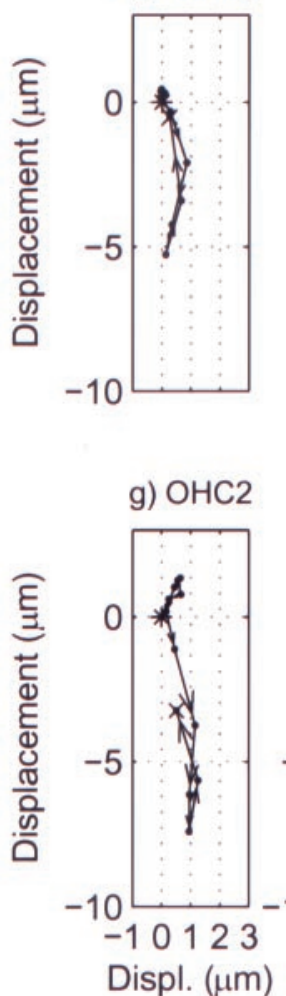

b) $\mathrm{OHC} 1$ top

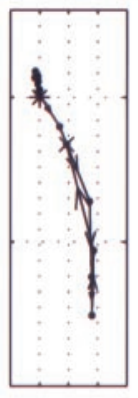

h) $\mathrm{OHC} 3$

c) $\mathrm{OHC} 2$ top

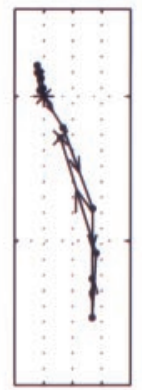

i) IP foot

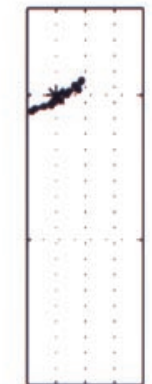

10

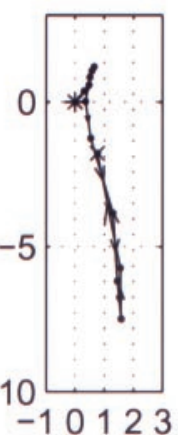

Displ. ( $\mu \mathrm{m})$

$-10123$ d) $\mathrm{OHC} 3$ top

e) IHC

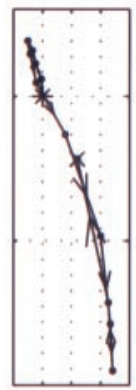

j) IP middle
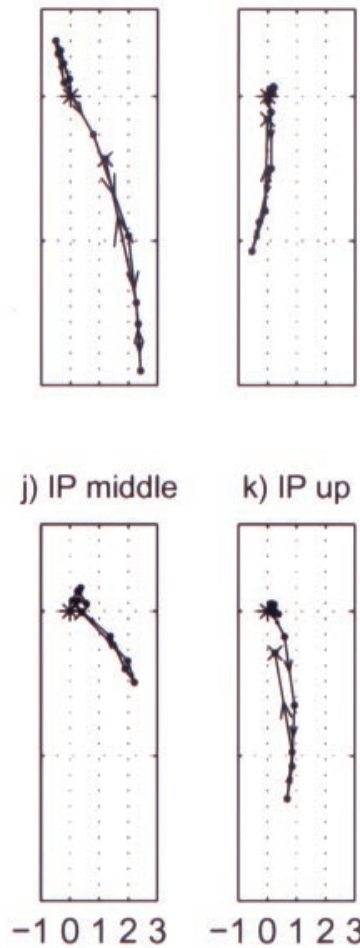

k) IP up

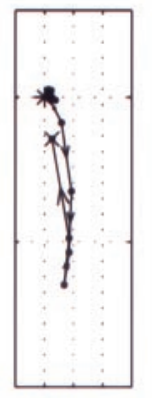

$-10123$

\section{f) $\mathrm{OHC} 1$}

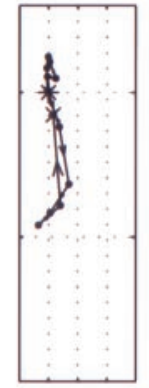

I) $\mathrm{OP}$ up

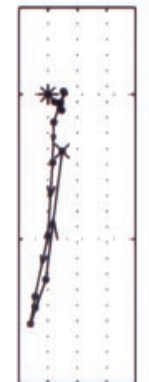

$-10123$

Displ. ( $\mu \mathrm{m})$

Figure 4. Top left, Confocal micrograph showing the difference in intensity values between the frames corresponding to the two maximum displacements of the cycle toward the scala vestibuli and toward the scala tympani, respectively. Bright and dark pixels correspond to significant displacements, whereas absence of motion appears in a medium green-gray color. The trajectories of different points along the reticular lamina $(a-d)$, on the hair cell bodies $(e-h)$, and on the inner and outer pillar cells $(i-l)$ are shown, as measured from the optical flow computation. Bottom, Details of optical flow trajectories of points on the reticular lamina $(a-d)$, at the cell bodies below $(e-h)$, and along the inner $(i-k)$ and outer $(l)$ pillar cells. The initial position (indicated by a star) was taken as the origin for each trajectory. The final position was marked by a $\times$ symbol. Note the large displacement in the negative direction (i.e., when the cochlear partition is biased toward the scala tympani) and the difference in orientation of the trajectories obtained at the bottom and top parts of the inner pillar cells. IHC, Inner hair cell; $O H C$, outer hair cell; IP, inner pillar cell; $O P$, outer pillar cell. Top right, Plot of the trajectory amplitudes in micrometers for 15 points along the reticular lamina (from the inner hair cell to the third-row outer hair cell) as a function of the distance from the inner hair cell apex. Note the clear linear growth of the amplitude, reflecting a rigid motion of the reticular lamina. The dashed line is a best linear fit to the set of data points. $R L$, Reticular lamina. 


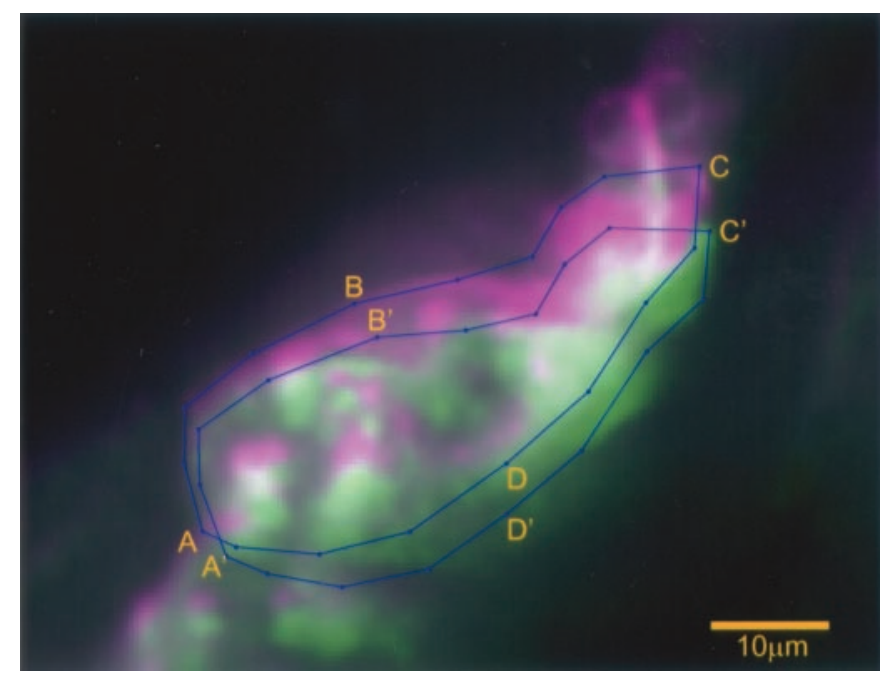

Figure 5. Deformations of the projected profile of the inner hair cell. A contour delimiting the profile of the cell was drawn by hand for the first frame of the sequence shown in Figure 4 and was left to evolve according to the measured displacements between successive frames. The image is a merged view of the two maximum displacements of the inner hair cell toward the scala vestibuli and scala tympani, respectively, with the corresponding profiles superimposed. Only the channel corresponding to the RH795 dye (Fig. 4A, red) is displayed. The length of the cell was measured as the distance from point $A$ to point $C$, and its width was measured as the distance from $B$ to $D$. $\left(A^{\prime}-D^{\prime}\right.$ are the corresponding points in the second frame.)

The reticular lamina moved as a stiff plate without noticeable deformation. Assuming a rigid rotation of the complex formed by the inner hair cell and the outer hair cells (Rhode and Geisler, 1966), one would expect the axis of rotation to be situated in the synaptic region near the base of the inner hair cell, because this region showed very little movement. The reticular lamina indeed appeared at a first approximation to move as a stiff plate, but the inner hair cell body could not be considered rigid. In the experiment shown in Figure 5 (same preparation as shown in Fig. 4), the length of the inner hair cell from base to apex (Fig. 5, distance $A-C$ ) first showed an increase of $0.81 \mu \mathrm{m}$, followed by a large 3.2 $\mu \mathrm{m}$ decrease, after which it increased back to its initial value. The changes in the cell width (Fig. $5 B-D$ ) were approximately four times smaller, implying that the cell underwent significant shape change. Because of the deformability of the inner hair cell, it is not possible to identify the motion of the reticular lamina, even when considered a perfectly stiff plate, with a rotation around a unique pivot axis. Nevertheless, it makes sense to define an effective pivot axis for the reticular lamina that depends on the pressure gradient applied, or equivalently on time in a periodic motion.

To estimate the location of this effective pivot axis from the optical flow data, the observed motion of the reticular lamina was compared with the predicted motion for a rotation around a given axis, subsequently minimizing the mean square error over the possible axis locations. In this way, we measured an average (rather than instantaneous) pivot axis, which depended on the set of time points retained in the estimation. In Figure 6, we show the pivot points estimated for two different experiments (Fig. 6A, $B$ ) and for three sets of time points (Fig. 6, 0, 1, and 2) corresponding to large, medium, and small angular displacements of the reticular lamina. For experiment A, the pivot points lie on the modiolar side of the inner hair cell. However, this was not always the case, as illustrated in Figure $6 B$. In both experiments, the location of the pivot axis showed a significant dependence on the amplitude of the displacement and was found consistently to lie closer to the apex of the inner hair cell at lower amplitudes. This dependency can be understood as a consequence of the deformability of the inner hair cell. If this cell were rigid, there would be little discrepancy between the pivot axes at small and large amplitudes. During sound stimulation, the displacements are smaller than those evoked by the scala tympani pressure changes. Thus, small angular displacement of the reticular lamina is probably the situation most relevant to events occurring during sound stimulation.

We also analyzed the positions of the pivot points for the outer hair cell bodies and the inner and outer pillar cells (Fig. 7). It was not possible to perform a very precise estimation, because errors on the optical flow map were more significant in these regions, and the estimated pivot points showed, not surprisingly, a larger variability than for the reticular lamina. However, the analysis clearly showed deformation occurring within the organ of Corti. In particular, the outer hair cell bodies did not rotate rigidly but rather showed distinct bending of their basal parts, manifest as a significant radial motion in the direction of the Hensen's cells for displacements of the basilar membrane toward the scala vestibuli (i.e., at positive pressures). It was also apparent that the inner and outer pillar cells followed different motion patterns. The inner pillar cell did not appear to be fully rigid, as reflected by the discrepancies in the pivot points estimated for the basal and apical parts of the cell. The outer pillar cell (of which only the top part was clearly visible) appeared to follow a motion pattern more similar to that of the first-row outer hair cell. Although the pivot points for structures showing a significant amount of deformation are inherently less informative, it is clear that the outer hair cell bodies do not have the same effective axis of rotation as other parts of the organ. The fact that different regions of the organ have different centers of rotation means that shearing motion occurs during basilar membrane displacement. Thus, such shearing motion occurs not only between the reticular lamina and the overlying tectorial membrane (Ulfendahl et al., 1995; Gummer et al., 1996) but also for structures sandwiched between the basilar membrane and the reticular lamina.

\section{DISCUSSION}

This study clarifies several important aspects of the passive mechanical properties of the hearing organ. These are an important determinant of the vibration of the hearing organ during sound stimulation. It is shown that under the present experimental conditions, (1) certain regions of the organ are more easily deformed than others, suggesting that different regions have different mechanical properties; (2) the reticular lamina behaves as a stiff plate with its center of rotation at the inner hair cell region; (3) the inner hair cell, despite being enclosed with supporting cells, cannot be considered structurally static and, together with the inner pillar cell, forms a region that is easily deformed; and (4) the basal poles of the outer hair cells show significant bending.

\section{Mechanical properties of the hearing organ}

The visualization of a cross section of the organ during basilar membrane displacement provides an opportunity to explore the mechanical properties of specific structures. Certain regions of the hearing organ thus appeared to have a higher stiffness than other regions, as judged from an apparent lack of structural deformation. Such stiffness variation has often been inferred from 
0
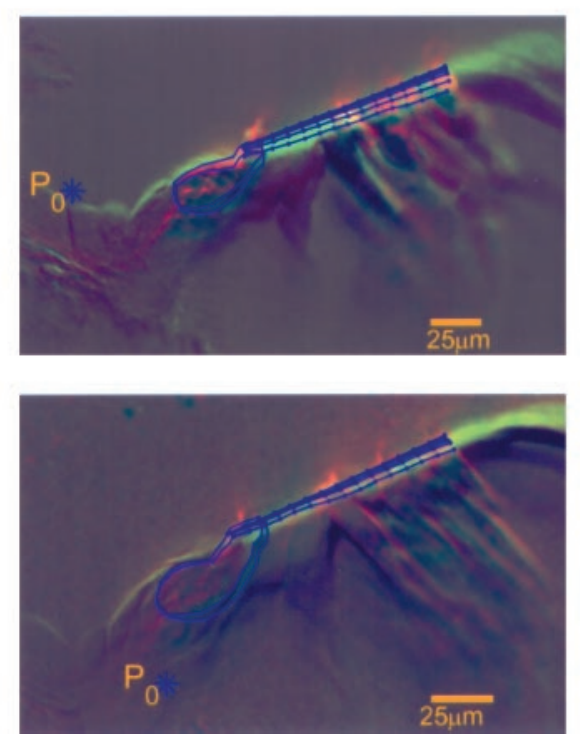

1
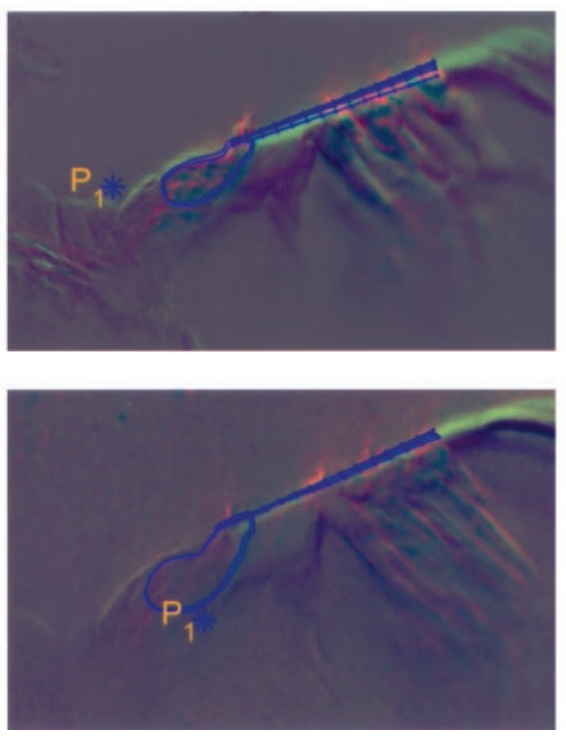

2
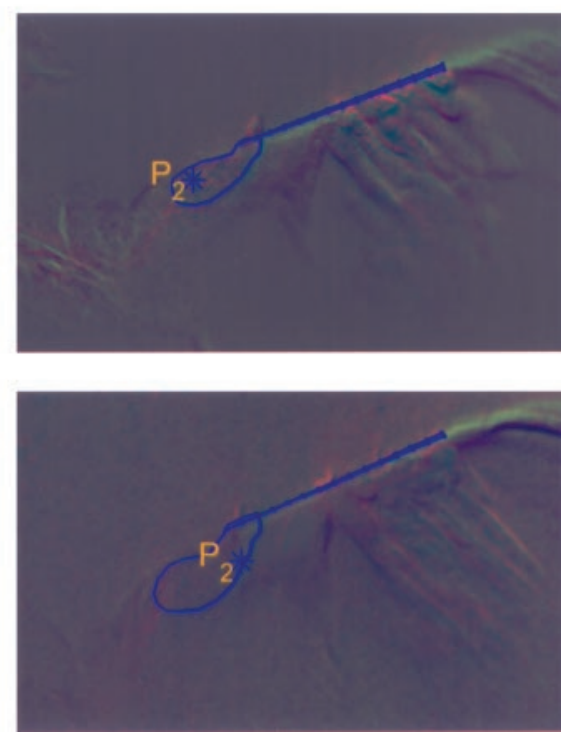

Figure 6. Determination of the effective pivot axis of the reticular lamina. Results are shown for two different experiments $(A, B)$. For each experiment, a least-squares estimation of the reticular lamina pivot axis was performed using three different series of time points, corresponding to different angular displacements of the reticular lamina: 0 , The full series of 13 frames (maximum angular displacements of 3.4 and $2.7^{\circ}$ for experiments $A$ and $B$, respectively); 1 , a medium subseries (maximum angular displacements of 3.0 and $1.9^{\circ}$ for $A$ and $B$, respectively); and 2, a small subseries (maximum angular displacements of 1.1 and $0.95^{\circ}$ for $A$ and $B$, respectively). Displayed in each case are the image difference between the two frames corresponding to the maximum displacements upward and downward, together with the estimated pivot axis, and a sample of gridlines showing intermediate positions of the reticular lamina. Note how the location of the pivot axis changes with angular displacement in each of the two experiments, moving toward the apical pole of the inner hair cell for smaller displacements. The profile of the inner hair cell is outlined in blue.

structural data [for a review of cochlear micromechanics, see Patuzzi (1996)]. Frequently, the inner hair cell region has been assigned a high compliance, because it is difficult to see how movement of the organ would otherwise be possible. This study provides experimental support for this common assumption, because the inner hair cell region showed reversible structural deformation. The inner pillar cells also appear to be easily deformed, whereas the outer pillars displayed rigid-body-type motion, at least the part of them that we were able to visualize. Such a stiffness distribution has also been suggested by studies of basilar membrane stiffness in living animals, in which the region of the basilar membrane underlying the outer pillar cells had a significantly higher stiffness than surrounding regions (Olson and Mountain, 1994).

\section{The reticular lamina pivot point}

In the present experiments, the reticular lamina behaved as a stiff plate, as suggested previously (von Békésy, 1960). The motion of the reticular lamina had characteristics similar to those found in experiments using sound stimulation (e.g., little motion of parts of the reticular lamina close to the center of the cochlea and a gradual increase when moving in the direction of the Hensen cells) (Khanna et al., 1989; Ulfendahl et al., 1996; Khanna and Hao, 1999; Hemmert et al., 2000b). This also carries the implication that the reticular lamina vibrates around a point located somewhere in the modiolar region of the organ. The present study extends these previous observations and allows the assignment of a pivot point for the reticular lamina. Interestingly, the location of this pivot point was dependent on the motion amplitude of the organ. For low amplitudes, the pivot point was consistently found closer to the apex of the inner hair cells than at high amplitudes. This dependence on stimulus amplitude appeared to be a consequence of the deformability of the inner hair cell and inner pillar

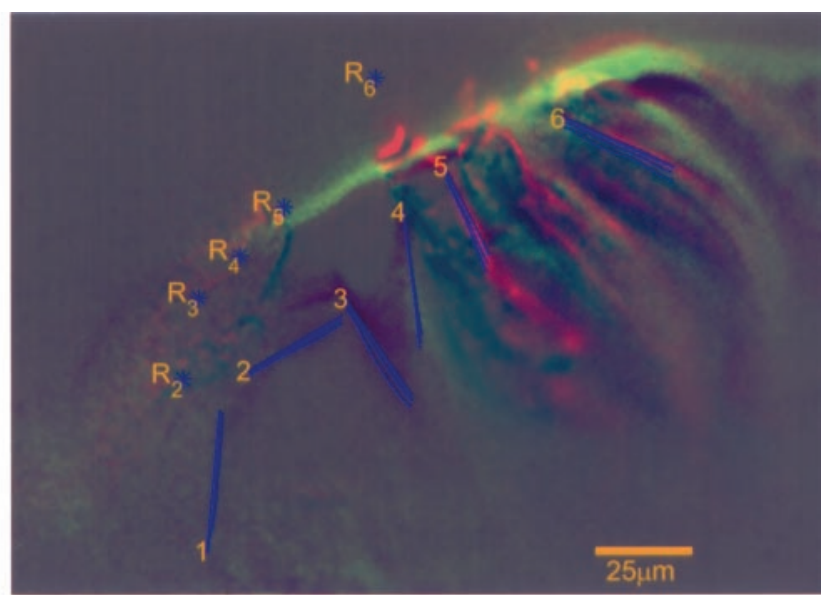

$\mathrm{R}_{1}$ *

Figure 7. Movement of different points along the pillar cells and sensory cells accompanying basilar membrane displacements. Pivot axes are shown for the inner and outer pillar cells and for the outer hair cells. The estimation was performed for a subseries of the full sequence corresponding to small angular displacements of the structures in the organ. (One of the pivot axes, $R_{1}$, was estimated outside the frame of the image, as shown by the asterisk.) The difference in the location of the pivot axes for the basal and apical parts of the inner pillar cell indicates cellular deformation. Note that because of the nonrigidity of the outer hair cell bases and of the inner pillar cell, different parts of these cells move around different instantaneous axes of rotation. Therefore, points $R_{1}-R_{6}$ actually represent averaged pivot axes for the corresponding lines highlighted on the cells. Points $R_{1}-R_{4}$ carry more information than points $R_{5}$ and $R_{6}$, however, because the inner and outer pillar cells were seen to be less amenable to deformation than the outer hair cell bodies. 
cell regions. If similar motion occurs during sound stimulation, as indeed appears to be the case, the pivot point should be located very close to the hair bundle of the inner hair cells. This is functionally interesting and implies that the motion of the reticular lamina may affect the inner hair cell stereocilia bundle directly.

\section{Shearing motion at the outer hair cells}

In the present study, the motion of the reticular lamina was relatively simple, consisting of a displacement essentially perpendicular to its long axis. There was no significant radial component, unlike previous studies in which point-like stimulation was used (Hu et al., 1999). However, the situation became increasingly complex for structures located between the basilar membrane and the reticular lamina. It was evident that position shifts of the organ toward the scala vestibuli were accompanied by lateral motion of the outer hair cells, in contrast to the classic rigid-body theory of cochlear motion (ter Kuile, 1900; Rhode and Geisler, 1966; Hemmert et al., 2000a).

Such lateral motion would be a natural consequence of the different centers of rotation for different structures (i.e., shearing movement inside the organ), but the inclination of the outer hair cells with respect to the basilar membrane may also contribute. The geometry and cellular attachments of the organ certainly allow bending of the cell bodies, as also reported previously for isolated cells stimulated by external electric fields (Frolenkov et al., 1997). This result is also in agreement with mathematical models showing that the outer hair cell soma has a relatively low bending stiffness (Spector et al., 1998, 2002), at least compared with the Deiters cells.

The amount of outer hair cell bending is clearly dependent on the stiffness of the cell, so a small change of stiffness could potentially modify the movement of the reticular lamina and stereocilia bundles by altering the effective mechanical coupling between the basilar membrane and the reticular lamina. Therefore, the outer hair cells are at an ideal position to influence organ of Corti micromechanics, either through a change in cell length (Brownell et al., 1985) or through an alteration of cellular stiffness (Chan et al., 1998; He and Dallos, 1999).

Shearing and bending forces may also have implications for outer hair cell motility, because these cells may be affected in several different ways by mechanical deformation (Canlon et al., 1988; Brundin et al., 1989; Brundin and Russell, 1994; Fridberger and Ulfendahl, 1996; Chan and Ulfendahl, 1999; Raphael et al., 2000; Santos-Sacchi and Rybalchenko, 2002). Consequently, these forces could induce motile responses, causing a direct modulation of organ of Corti vibration.

\section{Using an in vitro model to explore cochlear mechanics}

To achieve the necessary optical access, this study was performed using an in vitro preparation. This has some obvious shortcomings. For example, it has been shown that the mechanical properties of the hearing organ change slowly after the death of the animal (Olson and Mountain, 1994). In our case, the isolated preparation was oxygenated continuously, and it has been shown previously that the preparation sustains stable sound-evoked cochlear microphonic potentials for up to $5 \mathrm{hr}$ after the death of the animal (Ulfendahl et al., 1996). Moreover, many features of the sound-induced mechanical responses of this and similar preparations have been measured (Ulfendahl et al., 1996; Hemmert et al., 2000a,b) and subsequently confirmed in living animals (Cooper and Rhode, 1996; for review, see Ulfendahl, 1997; Khanna and
Hao, 1999; Zinn et al., 2000). Thus, substantial data indicate that the mechanical properties of the cells were normal, although the "cochlear amplifier" (for review, see Robles and Ruggero, 2001) probably does not function to the extent seen in the living animal.

It is the sound-induced alternating pressure gradient across the cochlear partition that causes mechanical stimulation of the sensory hair cells. In the intact inner ear, the pressure changes and resulting mechanical events are normally of very low amplitudes, with frequencies of $>20-30 \mathrm{~Hz}$. Here, the stimulus frequency was close to zero, and the resulting movement of the organ was larger than that found during sound stimulation. Because of the nature of the stimulus, altered cochlear amplification is not expected to have very much effect on the observations we present, and the changes in the cellular architecture may be considered the result of a passive process. However, because very little is known about sound-induced cellular events within the hearing organ, these observations give valuable information and clearly reveal important aspects of the basic motion pattern of the hearing organ. Moreover, such passive mechanical events will also clearly be important in an "active" cochlea operating under fully physiological conditions.

In summary, the motion pattern of the hearing organ is more complicated than reflected in most current models. The motion of internal structures of the organ showed significant radial and shearing components in addition to the transversal motion that was dominant at the reticular lamina. The outer hair cells are ideally positioned to actively modulate the mechanical coupling from the basilar membrane to the reticular lamina. Because the reticular lamina has its center of rotation at the inner hair cell, it may have a direct mechanical effect on the transduction process at the inner hair cell stereocilia bundle.

\section{REFERENCES}

Barron JL, Fleet DL, Beauchemin SS (1994) Performance of optical flow techniques. Int J Comput Vision 12:43-77.

Bernard C (2001) Discrete wavelet analysis for fast optic flow computation. Appl Comp Harmonic Anal 11:32-63.

Boutet de Monvel J, Le Calvez S, Ulfendahl M (2001) Image restoration for confocal microscopy: improving the limits of deconvolution, with application to the visualization of the mammalian hearing organ. Biophys J 80:2445-2470.

Brownell WE, Bader CR, Bertrand D, de Ribaupierre Y. (1985) Evoked mechanical responses of isolated cochlear outer hair cells. Science 227:194-196.

Brundin L, Russell I (1994) Tuned phasic and tonic motile responses of isolated outer hair cells to direct mechanical stimulation of the cell body. Hear Res 73:35-45.

Brundin L, Flock A, Canlon B (1989) Sound-induced motility of isolated cochlear outer hair cells is frequency-specific. Nature 342:814-816.

Canlon B, Brundin L, Flock Å (1988) Acoustic stimulation causes tonotopic alterations in the length of isolated outer hair cells from guinea pig hearing organ. Proc Natl Acad Sci USA 85:7033-7035.

Chan E, Ulfendahl M (1999) Mechanically-evoked shortening of outer hair cells isolated from the guinea pig organ of Corti. Hear Res 128:166-174.

Chan E, Suneson A, Ulfendahl M (1998) Acoustic trauma causes reversible stiffness changes in auditory sensory cells. Neuroscience 83:961-968.

Cooper NP, Rhode WS (1996) Fast travelling waves, slow traveling waves and their interactions in experimental studies of cochlear mechanics. Audiol Neurosci 2:289-299.

Flock Å, Strelioff D (1984) Studies on hair cells in isolated coils from guinea pig cochlea. Hear Res 15:11-18.

Flock A, Flock B, Fridberger A, Scarfone E, Ulfendahl M (1999) Supporting cells contribute to control of hearing sensitivity. J Neurosci 19:4498-4507.

Fridberger A, Ulfendahl M (1996) Acute mechanical overstimulation of isolated outer hair cells causes changes in intracellular calcium without shape changes. Acta Otolaryngol (Stockh) 116:17-24.

Fridberger A, van Maarseveen JTPW, Scarfone E, Ulfendahl M, Flock B, Flock A (1997) Pressure-induced basilar membrane position shifts and the stimulus-evoked potentials in the low-frequency region of the guinea pig cochlea. Acta Physiol Scand 161:239-252. 
Frolenkov GI, Kalinec F, Tavartkiladze GA, Kachar B (1997) Cochlear outer hair cell bending in an external electric field. Biophysics 73:1665-1672.

Gummer AW, Hemmert W, Zenner HP (1996) Resonant tectorial membrane: its crucial role in frequency tuning. Proc Natl Acad Sci USA 93:8727-8732.

He ZZ, Dallos P (1999) Somatic stiffness of cochlear outer hair cells is voltage-dependent. Proc Natl Acad Sci USA 96:8223-8228.

Hemmert W, Zenner HP, Gummer AW (2000a) Three-dimensional motion of the organ of Corti. Biophys J 78:2285-2297.

Hemmert W, Zenner HP, Gummer AW (2000b) Characteristics of the travelling wave in the low-frequency region of a temporal bone preparation of the guinea-pig cochlea. Hear Res 142:184-202.

Horn BKP, Schunck BG (1981) Determining optical flow. Artif Intell 17:185-203.

Hu X, Evans BN, Dallos P (1999) Direct visualization of organ of Corti kinematics in a hemicochlea. J Neurophysiol 82:2798-2807.

Khanna SM, Hao LF (1999) Nonlinearity in the apical turn of the living guinea pig cochlea. Hear Res 135:89-104.

Khanna SM, Flock A, Ulfendahl M (1989) Changes in cellular tuning along the radial axis of the cochlea. Acta Otolaryngol Suppl (Stockh) 467:163-173.

Naidu RC, Mountain DC (1998) Measurements of the stiffness map challenge a basic tenet of cochlear theories. Hear Res 124:124-131.

Olson ES, Mountain DC (1994) Mapping the cochlear partition's stiffness to its cellular architecture. J Acoust Soc Am 95:395-400.

Patuzzi R (1996) Cochlear micromechanics and macromechanics. In: The cochlea (Dallos P, Popper AN, Fay RR, eds), pp 186-257. New York: Springer.

Raphael RM, Popel AS, Brownell WE (2000) A membrane bending model of outer hair cell electromotility. Biophys J 78:2844-2862.

Rhode WS, Geisler CD (1966) Model of the displacement between opposing points on the tectorial membrane and reticular lamina. J Acoust Soc Am 42:185-190.

Robles L, Ruggero MA (2001) Mechanics of the mammalian cochlea. Physiol Rev 81:1305-1352.

Russell IJ, Schauz C (1995) Salicylate ototoxicity: effects on the stiffness and electromotility of outer hair cells isolated from the guinea pig cochlea. Audiol Neurosci 1:309-320.
Santos-Sacchi J, Rybalchenko V (2002) Tension-dependent chloride current affects OHC capacitance. Assoc Res Otolaryngol Midwinter Meeting 2002, St. Petersburg Beach, FL, January. Abstract 969.

Spector AA, Brownell WE, Popel AS (1998) Estimation of elastic moduli and bending stiffness of the anisotropic outer hair cell wall. J Acoust Soc Am 103:1007-1012.

Spector AA, Ameen M, Schmiedt RA (2002) Modeling 3-D deformation of outer hair cells and their production of active force in the cochlea. Biomech Model Mechanobiol 1:1-13.

ter Kuile E (1900) Die Übertragung der Energie von der Grundmembran auf die Haarzelle. Pflügers Arch 79:146-157.

Tolomeo JA, Holley MC (1997) Mechanics of microtubule bundles in pillar cells from the inner ear. Biophys J 73:2241-2247.

Tolomeo JA, Steele CR, Holley MC (1996) Mechanical properties of the lateral cortex of mammalian auditory outer hair cells. Biophys J $71: 421-429$.

Ulfendahl M (1997) Mechanical responses of the mammalian cochlea. Prog Neurobiol 53:331-380.

Ulfendahl M, Khanna SM, Heneghan C (1995) Shearing motion in the hearing organ measured by confocal laser heterodyne interferometry. NeuroReport 6:1157-1160.

Ulfendahl M, Khanna SM, Fridberger A, Flock ^, Flock B, Jäger W (1996) Mechanical response characteristics of the hearing organ in the low-frequency regions of the cochlea. J Neurophysiol 76:3850-3862

Ulfendahl M, Chan E, McConnaughey WB, Prost-Domasky S, Elson EL (1998) Axial and transverse stiffness measures of cochlear outer hair cells suggest a common mechanical basis. Pflügers Arch 436:9-15.

Ulfendahl M, Scarfone E, Flock A, Le Calvez S, Conradi P (2000) Perilymphatic fluid compartments and intercellular spaces of the inner ear and organ of Corti. NeuroImage 12:307-313.

Voie AH, Burns DH, Spelman FA (1993) Orthogonal plane fluorescence optical sectioning: three dimensional imaging of macroscopic biological specimens. J Microsc 170:229-236.

von Békésy G (1960) Experiments in hearing. New York: McGraw-Hill.

Zinn C, Maier H, Zenner H-P, Gummer AW (2000) Evidence for active, nonlinear, negative feedback in the vibration response of the apical region of the in-vivo guinea-pig cochlea. Hear Res 142:159-183.

Zwislocki JJ, Cefaratti LK (1989) Tectorial membrane II: stiffness measurements in vivo. Hear Res 42:211-228. 